\title{
Benefits of cardiac rehabilitation in coronary heart disease
}

\begin{abstract}
Cardiovascular diseases are the leading cause of death and an important cause of morbidity. Cardiac rehabilitation is a major part of secondary prevention for patients with coronary heart disease with several benefits across its spectrum, including reductions in mortality, cardiac events and improvement in quality of life, among patients after myocardial infarction percutaneous coronary intervention and coronary artery bypass graft. This review sought to present several evidence-based benefits of cardiac rehabilitation in the coronary heart disease scenarios of secondary prevention.
\end{abstract}

Volume 2 Issue 4 - 2017

\author{
Rodrigo de Moura Joaquim,' Rafael \\ Fernandes Martins,' Ciro Teixeira Campos ${ }^{2}$ \\ 'Instituto Dante Pazzanese de Cardiologia, Brazil \\ ${ }^{2}$ Imperial Hospital de Caridade, Brazil
}

Correspondence: Rodrigo de Moura Joaquim, Instituto Dante Pazzanese de Cardiologia, São Paulo, Brazil, Tel +55 (48) 988251619, Email rodrigojoaquim@gmail.com

Received: November 19, 2017 | Published: December 08, 2017

\section{Introduction}

Cardiovascular (CV) diseases are still the leading cause of death and also an important cause of physical incapacity and disability, significantly increasing health costs. In addition, numbers of people living longer with symptomatic coronary heart disease (CHD) are rising. CHD is estimated affect $7 \%$ of the population, with $8,3 \%$ of men and $6,1 \%$ of women. Each year about 785000 Americans will suffer a new myocardial infarction (MI) and nearly 470000 will have a recurrent one. Accessibility of health services for people with CHD preventing secondary events, remain an important issue nowadays. ${ }^{1-5}$

Cardiac rehabilitation (CR) programs are recognized as important part of secondary prevention care of CHD patients, including benefits in $\mathrm{CV}$ mortality and morbidity, adherence to preventive therapies and risk factor control, psychological and physiological benefits of exercise training and improvement in quality of life (QOL). It has been given a Class I recommendation, from the American Heart Association, the American College of Cardiology the European Society of Cardiology and the Brazilian Society of Cardiology, with exercise therapy consistently identified as a central element. ${ }^{1-4,6}$

Although the first meta-analysis and systematic reviews of randomized control trials (RCT) showed a $25 \%$ to $40 \%$ reduction in all-cause and cardiovascular mortality, those studies were published nearly 30 years ago. ${ }^{7,8}$ With advances in CHD medical treatment in the past two decades, the possible benefits of mortality reduction, from exercise-based CR compared to usual care alone, were deemed as attenuated and possibly null by those new therapies, generating doubts about the role of CR. ${ }^{9}$ This review sought to evaluate aspects of $\mathrm{CR}$ regarding general CHD patients and its different scenarios and subgroups, evaluating the actual impact of CR for secondary prevention in clinical practice.

\section{Coronary heart disease}

Almost 30 years ago, the first systematic reviews of RCTs showed comprehensive benefit of $\mathrm{CR}$ for secondary prevention in clinical practice, in a time with small trials and only one trial showing benefit in isolation. Oldridge et al. ${ }^{7}$ carried out a meta-analysis of RCTs that included 4,347 patients, with 2,202 patients in the CR group.
In that analysis a reduction of $24 \%$ in all-cause death (Odds Ratio [OR]:0.76;95\% Confidence Interval [CI]:0.63-0.92) and 25\% in cardiac death (OR:0.75;95\% CI:0.62-0.93) was found. A year later O'Connor et al. ${ }^{8}$ published an analysis of $22 \mathrm{RCTs}$, with 4,554 patients including 2,310 participating in CR programs. After an average of 3 years follow-up, a reduction in all-cause mortality and CV mortality of 20\% (OR:0.80;95\% CI:0.66-0.96) and 22\% (OR:0.78;95\% CI:0.63$0.96)$ respectively, was found, with greater benefits in the first year including reduction in sudden cardiac death.

The RAMIT trial ${ }^{10}$ (Rehabilitation after myocardial infarction trial: multi-centre randomized controlled trial of comprehensive cardiac rehabilitation in patients following acute myocardial infarction), evaluated 1813 post-MI patients in England and Wales, found no difference in all-cause mortality at 2 years (Relative Risk [RR]:0.98;95\% CI:0.74-1.30) and after a extended follow-up of 7-9 years (RR:0.99;95\% CI:0.85-1.15). It is noteworthy that this study reached lower than estimated total number of patients with exclusions of over $30 \%$ of the evaluated sample and was therefore underpowered to show any end-point differences alone. Recently a meta-analysis of 63 studies, that included the RAMIT trial with 14.486 patients, included old and recent trials. With a median follow-up of 12 months, no difference in all-cause mortality was found (RR:0.96;95\% CI:0.881.04), but reductions in CV mortality (RR:0.74;95\% CI:0.64-0.86) and risk of hospital admission (RR:0.82;95\% CI:0.70-0.96) were present when they compared CR patients to the control group. The lack of benefit in mortality may be explained by a more mixed population in recent studies, including possibly very low risk populations like patients with only angina and non-obstructive CHD as defined by angiography and because they were conducted in the era of optimal medical therapy and newer techniques of revascularization for CHD although the reduction in CV mortality was maintained. The authors concluded that with these benefits and improvement in quality of life, recommendations of $\mathrm{CR}$ in patients with established CHD should be maintained. ${ }^{9}$ Unanimously neither of those systematic reviews found difference in $\mathrm{MI}$ and need for new revascularization in patients that underwent $\mathrm{CR}$ compared to those who did not. ${ }^{7-9}$ Figure 1 resumes the overall evidence-based benefits found in CHD patients who undergo CR generally and in specific scenarios. 


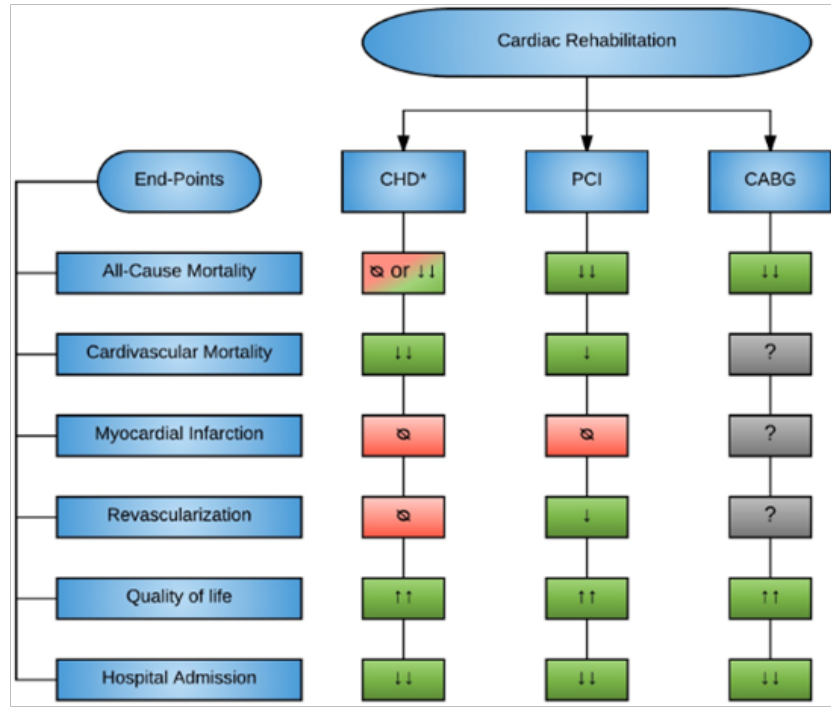

Figure I Different end-points influenced by cardiac rehabilitation in coronary heart disease.

Note: $\mathrm{CHD}$, coronary heart disease; $\mathrm{PCl}$, percutaneous coronary intervention; $\mathrm{CABG}$, coronary artery bypass graft

*Includes post-myocardial infarction patients its benefits in mortality seems to exist and be of significance depending in the chosen population. Q: No apparently benefit; ?: Not sufficient evidence to support benefit position; $\downarrow$ Benefit proved with small body of evidence; $\downarrow \downarrow$ or $\uparrow \uparrow$ : Benefit proved with strong body of evidence

It is important to know that dose of CR was not accounted in those analysis. Pio et al. ${ }^{11}$ found that CR dose may play an important role regarding its benefits. In a meta-analysis they found that moderate dose (12-35CRsessions) and higher dose ( $\geq 36 \mathrm{CR}$ sessions) had a reduction in all-cause mortality of $42 \%$ to $44 \%(\mathrm{p}<0.001)$ as compared to a control group and a low dose $\mathrm{CR}(<12 \mathrm{CR}$ sessions $)$. They also found that higher dose may be associated with fewer percutaneous coronary interventions (PCI) (RR:0.65;95\% CI:0.50-0.84). Thus it is important not only to select a higher risk patient but also to maintain that patient in a CR program, with an adequate time of exercise, to see the potential benefits of CR.

\section{Percutaneous coronary intervention}

Despite CR benefits being demonstrated in post-MI patients, less is known of CR benefits after PCI, but the trending seems to be the same. The ETICA trial ${ }^{12}$ (Exercise training intervention after coronary angioplasty) who evaluated 118 patients, half in an exercise training group, found improvements in QOL and functional capacity in the exercise training group, with a reduction in cardiac events, accounting for MI and new revascularization (11.9versus 32.2\%; RR:0.71;95\% CI:0.60-0.91; $\mathrm{p}=0.008$ ), and also a reduction in hospital admission (18.6 versus 46\%; RR:0.69;95\% CI:0.55-0.93;p<0.001) with moderate training.

Goel et al. ${ }^{13}$ performed a retrospective analysis of data from a prospectively collected registry of 2395 patients who realized PCI from 1994 to 2008 in the United States, where 40\% (964) engaged CR. Within a median follow-up of 6.3 years, and using different statistical analysis methods, a reduction in all-cause mortality of $45 \%$ to $47 \%$ was found $(p<0,001)$ in those patients that performed CR. compared with those who did not engage $\mathrm{CR}$. The $\mathrm{CR}$ patients also experienced a reduction in CV mortality up to $39 \%$ but that didn't meet statistical significance. There was no difference regarding the occurrence of myocardial infarction or the need to repeat revascularization. In this study the number necessary to treat (NNT) to prevent one death was 34 in the first year and 22 after 5 years. Another study, with a expanded analysis and a median follow-up of 8 years, found that $\mathrm{CR}$ was still associated with reduction in all-cause mortality (Hazard Ratio(HR):0.67; 95\% CI:0.55-0.82;p<0.001) and in CV mortality(HR:0.67;95\% CI;0.47-0.95;p=0.024) on direct and matchpair analysis. The benefits on all-cause mortality were maintained in the higher CHD risk diabetic patients (HR:0.56;95\% CI:0.39$0.80 ; \mathrm{p}=0.002$ ) of that study. ${ }^{14}$

\section{Coronary artery bypass graft}

In a community-based analysis from 1996 to 2007, Pack et al. ${ }^{15}$ evaluated the association between CR attendance and all-cause mortality adjusted for the propensity to attend CR. In 846 patients, $76 \%$ men with mean age of 66 years, who survived the first six months of surgery, 582(69\%) attended CR. During a mean follow-up of 9 years, CR participation was associated with a risk reduction in allcause mortality of $46 \%(\mathrm{HR}=0.54 ; 95 \% \mathrm{CI}: 0.40-0.74 ; \mathrm{p}<0.001)$ and a absolute risk reduction of $12,7 \%$ for all-cause mortality, with a NNT to prevent one death of 8 .

Smaller studies have also shown some benefits of CR in patients after CABG. In four months follow-up, Aronov et al. ${ }^{16}$ found improvement of $32.6 \%$ in exercise duration compared to baseline in CR patients and after one year, there was a reduction in CV adverse events $(11.1 \%$ versus $39.2 \%)$ when compared to the control group. Hedbäck et al. ${ }^{17}$ found a $54 \%$ reduction in all-cause mortality but this did not meet statistical significance $(\mathrm{p}=0.06)$, likely because of the small sample size, even with nearly ten years follow-up, but they found a reduction in $\mathrm{CV}$ adverse events a combined of $\mathrm{CV}$ death, $\mathrm{MI}$ and revascularization $(18.4 \%$ versus $34.7 \% ; \mathrm{p}<0.01)$, and also showed a reduction in hospital admissions $(\mathrm{p}<0.01)$. Another study with 149 patients in the CR group and 89 in the control group found a reduction in $\mathrm{CV}$ events after a two years follow-up $(4.7 \%$ vs. $14.0 \%, \mathrm{P}<0.05) .{ }^{18}$ There are no RCTs that have specifically tested CR in CABG, with an expressive number of patients, and despite those results being promising, those small studies are only hypothesis generators and no definitive conclusions can be draw regarding $\mathrm{CV}$ mortality and morbidity in the CABG subgroup (Figure 1).

\section{Diebetes mellitus}

Studies that evaluated exercise capacity by peak oxygen uptake, peak workload, heart rate, exercise duration, anaerobic threshold and metabolic equivalent tasks (METs) showed worst results in patients with diabetes mellitus (DM) leading to a lower improvement in exercise capacity, potentially diminishing the benefit of $\mathrm{CR}$ on that population. ${ }^{19-21}$ Metabolic and myopathic limitations associated with hyperglycemia are suggested as possible mechanisms for a lower improvement of exercise capacity. ${ }^{21}$

Recently Armstrong et al. ${ }^{22}$ evaluated a cohort of 13,158 patients with and without DM. Over a mean follow-up of 6.6 years, those who completed the program had a reduction in all-cause mortality (HR:0.46;95\% CI:0.37-0.56) and hospital admissions (HR:0.86;95\% CI:0.76-0.96), similar to outcomes of those without DM. A study with 700 post PCI patients with $\mathrm{DM}^{14}$ found after a propensity match analysis, that $\mathrm{CR}$ was associated with reduction in all-cause 
mortality and in the composite end-point of mortality, MI and need for revascularization. Patients with DM were also less likely to participated in CR (38\% versus $45 \%$; $\mathrm{p}=0.004)^{14}$ and to complete a CR program $(41 \% \text { versus } 56 \% ; \mathrm{p}<0.001)^{22}$ than those without DM. Those data support that despite a lower exercise capacity achieved in patients with DM after CR, the clinical benefit is maintained in this subgroup and a great effort of engaging those patients in $\mathrm{CR}$ programs need to be made.

\section{Older age}

In a Medicare beneficiary's analysis of 601,099 patients, who were $>65$ years old, $12.2 \%$ were in CR programs. A match-pair analysis was performed and for 70,040 patients a match was found. In those, cumulative mortality was lower in the $\mathrm{CR}$ group as compared to the control group, with mortality rates of $2.2 \%$ versus $5.3 \%$ in the first year and $16.3 \%$ versus $24.6 \%$ at 5 years $(\mathrm{p}<0.001)$. Those numbers indicate a relative reduction in mortality of $58 \%$ and $34 \%$ in the first and fifth year respectively. The reduction was greater as older the patients were in those with higher dose of exercise, in women and in patients with more comorbidities such as previous MI and coexisting heart failure. ${ }^{23}$ This suggests that the benefits and safety of CR, are the same in older as in younger patients and age should not be an issue for CR participation. Despite that most studies exclude older patients and a study found that CR engagement decreases by $1.2 \%$ per additional year of patient age. ${ }^{8,24}$

\section{Quality of life}

QOL, as assessed by validated questionnaires, generally shows improvement through trials comparing $\mathrm{CR}$ and a control group, including home-based, computer-based or traditional programs, also with benefits in anxiety and depression scales. ${ }^{12,25-28}$ In a recent postCABG study, Dabek et al. ${ }^{28}$ showed a improvement of more than 50\% in anxiety scales and symptoms such as dyspnea and chest pain in the $\mathrm{CR}$ group compared to a control group, with improvement in QOL $(p<0.01)$. In that study $66 \%$ of the patients though that participating in CR program helped then recover faster from surgery. The ETICA Trial. $^{12}$ (Exercise training intervention after coronary angioplasty) showed improvement in two QOF questionnaires in follow-up of six and twelve months with higher improve in patients who increased $>1$ MET in functional capacity. Anderson et al. ${ }^{9}$ reported in their metaanalysis of $63 \mathrm{RCTs}$, a better QOL in the group of CR in comparison to the control group, through different scenarios of $\mathrm{CHD}$, including post-MI and post-revascularization patients.

\section{Participation in CR programs}

Even after the first studies and analysis showed the potential benefits of CR, several challenges remained in implementation of CR programs and patient adherence to those. It's being consistent through all studies, older or recent, that only about one third of patients are referred and engage on a CR program for secondary prevention in the following months of an event, with that percentage being even lower in emerging countries. ${ }^{4,7-9}$ Even when as low as one outpatient CR session was needed for the patient to be considered part of the program Goel et al. ${ }^{6}$ found that only 964 of 2,395(40\%) did perform CR. Several factors are associated with low initiation and adherence to the programs, including socio-demographic factors, such as age, sex and ethnicity, patient medical condition and risk factors, including total number of comorbidities and service level factors can influence patient engagement. ${ }^{24}$
Regarding the RCTs itself, several of them are of small inclusion, recruitment of low-risk, middle-aged patients, with great variability in type of exercise and orientations, and involving mostly only men or a small number of women..$^{7-10}$ therefore being their findings generalized to a broader population, leading to mistrust in their benefits and reproducibility by other physicians and services, when applied to their daily practice. Specifically in women, where the benefits are consistently the same as in men they remain of low representative in most studies, with only $15 \%$ to $25 \%$, presenting themselves with more risk factors and probably more severe $\mathrm{CHD}^{9,24,29,30}$ One study found that the probability of women engaging in CR program was $7.1 \%$ lower than men. ${ }^{24}$

In a study with 3,871 patient, where $1,49739 \%$ ) engaged CR program and 1,193 completed it, the chance of completing CR program after primary PCI for acute MI was evaluated. Sunamura et al. ${ }^{29}$ found that elderly, female and low socioeconomic status patients were at increased risk of non-participation and non-completion of CR. They also found that patients with diabetes, current smokers and with history of prior MI were at risk of not completing the program. DM patients, despite the higher CHD risk, are also known to participate less in CR programs than patients without DM. ${ }^{14,22}$ Cultural and social believes, work conditions and house management while the patient is attending the program are associated with non-enrollment in CR programs. Factors regarding the programs itself such as enrollment burocracy, program viability in non-urban and low-income areas and programs that are mainly of one gender attendance or without a professional with knowledge of CHD, are also associated with the patient not being referred or enrolled in CR programs., ${ }^{2,31-33}$ Table 1 summarizes several factors associated with referring and engaging in CR programs. It's very important to know those limitations try to adapt and encourage patients to engage and stay in those programs.

Table I Factors associated with limited referral and engagement in CR programs

\begin{tabular}{l}
\hline Patient and social factors \\
\hline Female sex \\
Older Age \\
Limited health care insurance \\
Low socioeconomic status \\
Cultural believes \\
Limited social support \\
Home and work responsibilities \\
Medical factors \\
Multiple comorbidities \\
Diabetes mellitus \\
Depression \\
Musculoskeletal conditions \\
Healthcare system factors \\
Lack of referral and limited enrollment facilitation \\
Program availability and characteristics
\end{tabular}


Strategies to facilitate enrollment in CR programs include referring at hospital discharge plan, active follow up after a cardiac event and providing transportation and social assistance when required. ${ }^{2431}$ There is also evidence suggesting that participation in traditional CR program can be increased by as much as $18 \%$ to $30 \%$ with the use of multifaceted patient-targeted strategies, hence motivational communications delivered through letters, telephone calls, and home visits. ${ }^{34}$ Furthermore a Cochrane systematic review of data from 12 randomized controlled trials, with 1938 participants, in 6 different countries evaluated the effectiveness of home-based CR with supervised center-based CR, showing no difference in events after short term (12 months) and long term (24 months) follow-ups. Although acknowledging that further studies are needed the authors concluded that this is a viable option for patient personal preference and may affect favorably participation rates in CR programs. ${ }^{35}$

\section{Cost-effectiveness}

There are some controversies about how CR programs affect health care costs. Because of the bias of each countries health system, it is hard to generalize results to all, but CR programs usually have no effect or reduce total costs, with some studies showing that CR can be even cost saving, because it not only decreases costs but also increases survival. Ades et al..$^{36}$ analyzed the cost/benefit ratio of 21 months of CR, and found savings of USD 739 and Oldrige et al. ${ }^{37}$ evidenced savings of USD 9,200, over a period of 12 months in comparison with the control group.

Although two studies showed no difference in total health care costs between CR and control groups. ${ }^{38,39}$ when analyzed the costeffectiveness quality-adjusted life-year, one of them had an additional U.S. $\$ 42,535^{38}$ and the other a reduction of U.S. $\$ 650^{39}$ for CR compared to a control group. Aditional costs may be affected by local bias considering that a reduction in cardiovascular mortality and improving quality of life may positively impact on it.

\section{Conclusion}

This review supports the use of CR for secondary prevention, in patients with CHD and in its different scenarios, as a Class I indication, although acknowledging that data from RCTs involving a greater number and higher risk patients would be important to consolidate it even more. Future perspectives should also focus in patient selection, inclusion and adherence to $\mathrm{CR}$ in order to increase the number of benefited from the programs.

\section{Acknowledgements}

The statement is certify that all authors have seen and approed the manuscript being submited, have contributed significantely to the work, attest to the validity and ligitimacy of the data and its interpretation and agree to its submission to the International Physical Medicine \& Rehabilitation Journal. On be-half all Co-authors the corresponding author shallbear full responsibilty for the submission.

We attest that the article is the Authors Original work has not received prior publication and is not under consideration for publication elsewhere.

\section{Conflict of interest}

There is no conflict of interest regarding any of the authors and this paper and there was relationship with industry or other financial support fot this paper.

\section{References}

1. Roger VL, Go AS, Lloyd JDM, et al. Heart disease and stroke statistics-2011 update: A report from the american heart association. Circulation. 2011;123(4):e18-209.

2. Leon AS, Franklin BA, Costa F, et al. Cardiac rehabilitation and secondary prevention of coronary heart disease: an American heart association scientific statement from the council on clinical cardiology (subcommittee on exercise, cardiac rehabilitation, and prevention) and the council on nutrition, physical activity, and metabolism (subcommittee on physical activity), in collaboration with the american association of cardiovascular and pulmonary rehabilitation. Circulation. 2005;111(3):369-376.

3. Smith SC, Benjamin EJ, Bonow RO, et al. AHA/ACCF secondary prevention and risk reduction therapy for patients with coronary and other atherosclerotic vascular disease: 2011 update: a guideline from the American Heart Association and American College of Cardiology Foundation. J Am Coll Cardiol. 2011;124:2458-2473.

4. Herdy AH, López JF, Terzic CP, et al. South american guidelines for cardiovascular disease prevention and rehabilitation. Arq Bras Cardiol. 2014;103(2 Suppl 1):1-31.

5. Perk J, Backer DG, Gohlke H, et al. European guidelines on cardiovascular disease prevention in clinical practice. The fifth joint task force of the european society of cardiology and other societies on cardiovascular disease prevention in clinical practice (constituted by representatives of nine societies and by invited experts). Eur Heart J. 2012;33(13):1635-1701.

6. Goel K, Lennon RJ, Tilbury RT, et al. Impact of cardiac rehabilitation on mortality and cardiovascular events after Percutaneous coronary intervention in the community. Circulation. 2011;123:2344-2352.

7. Oldridge NB, Guyatt GH, Fischer ME, et al. Cardiac rehabilitation after myocardial infarction. Combined experience of randomized clinical trials. JAMA. 1988;260(7):945-950.

8. O'Connor GT, Buring JE, Yusuf S, et al. An Overview of randomized trials of rehabilitation with exercise after myocardial infarction. Circulation. 1989;80(2):234-244

9. Anderson L, Oldridge N, Thompson DR, et al. Exercise-Based cardiac rehabilitation for coronary heart disease. Cochrane systematic review and meta-analysis. J Am Coll Cardiol. 2016;67(1):1-12.

10. West RR, Jones DA, Henderson AH. Rehabilitation after myocardial infarction trial (RAMIT): multi-centre randomised controlled trial of comprehensive cardiac rehabilitation in patients following acute myocardial infarction. Heart. 2012;98(8):637-644.

11. Pio CSA, Marzolini S, Pakosh M, et al. Effect of cardiac rehabilitation dose on mortality and morbidity: a systematic review and meta-regression analysis. Mayo Clin Proc. 2017;92(11):1644-1659.

12. Belardinelli R, Paolini I, Cianci G, et al. Exercise training intervention after coronary angioplasty: the ETICA trial. J Am Coll Cardiol. 2001;37(7):1891-1900.

13. Goel K, Lennon RJ, Tilbury RT, et al. Impact of cardiac rehabilitation on mortality and cardiovascular events after percutaneous coronary intervention in the community. Circulation. 2011;123:2344-2352.

14. Jiménez NMF, LopezJF, Pérez BLM, , et al. Benefits of cardiac rehabilitation on cardiovascular outcomes in patients with diabetes mellitus after percutaneous coronary intervention. $J$ Am Heart Assoc. 2017;6(10):e006404.

15. Pack QR, Goel K, Lahr BD, et al. Participation in cardiac rehabilitation and survival after coronary artery bypass graft surgery: a communitybased study. Circulation. 2013;128(6):590-597. 
16. Aronov DM, Bubnova MG, Ioseliani DG, et al. The complex program of rehabilitation of patients with ischemic heart disease after coronary artery bypass surgery in ambulatory cardiorehabilitational department: Clinica effects of third stage of rehabilitation. Kardiologiia. 2017;57(3):10-19.

17. Hedbäck B, Perk J, Hörnblad M, et al. Cardiac rehabilitation after coronary artery bypass surgery: 10-year results on mortality, morbidity and readmissions to hospital. J Cardiovasc Risk. 2001;8(3)153-158.

18. Hansen D, Dendale P, Leenders M, et al. Reduction of cardiovascular event rate: different effects of cardiac rehabilitation in $\mathrm{CABG}$ and $\mathrm{PCI}$ patients. Acta Cardiol. 2009;64(5):639-644.

19. Suresh V, Harrison RA, Houghton P, et al. Standard cardiac rehabilitation is less effective for diabetics. Int J Clin Pract. 2001;55(7):445-448.

20. Kim HJ, Joo MC, Noh SE, et al. Long-Term outcomes of cardiac rehabilitation in diabetic and non-diabetic patients with myocardial infarction. Ann Rehabil Med. 2015;39(6):853-862.

21. Verges B, Patois VB, Cohen M, et al. Effects of cardiac rehabilitation on exercise capacity in type 2 diabetic patients with coronary artery disease. Diabet Med. 2004;21(8):889-895.

22. Armstrong MJ, Sigal RJ, Arena R, et al. Cardiac rehabilitation completion is associated with reduced mortality in patients with diabetes and coronary artery disease. Diabetologia. 2015;58(4):691-698.

23. Suaya JA, Stason WB, Ades PA, et al. Cardiac rehabilitation and survival in older coronary patients. J Am Coll Cardiol. 2009;54(1):25-33.

24. Al Quait A, Doherty P, Gutacker N, et al. In the modern era of percutaneous coronary intervention: Is cardiac rehabilitation engagement purely a patient or a service level decision? European Journal of Preventive Cardiology. 2017;24(13):1351-1357.

25. Houle J, Doyon O, Vadeboncoeur N, et al. Effectiveness of a pedometerbased program using a socio-cognitive intervention on physical activity and quality of life in a setting of cardiac rehabilitation. Can J Cardiol. 2012;28(1):27-32.

26. Mutwalli HA, Fallows SJ, Arnous AA, et al. Randomized controlled evaluation shows the effectiveness of a home-based cardiac rehabilitation program. Saudi Med J. 2012;33(2):152-159.

27. Reid RD, Morrin LI, Beaton LJ, et al. Randomized trial of an internetbased computertailored expert system for physical activity in patients with heart disease. Eur J Prev Cardiol. 2012;19(6):1357-1364.

28. Dąbek J, Pyka E, Piotrkowicz J, et al. Impact of post-hospital cardiac rehabilitation on the quality of life of patients after surgical treatment for coronary artery disease. Kardiochir Torakochirurgia Pol. 2017;14(2):120-126.
29. Sunamura M, Hoeve TN, Geleijnse ML, et al. Cardiac rehabilitation in patients who underwent primary percutaneous coronary intervention for acute myocardial infarction: determinants of programme participation and completion. Neth Heart J. 2017;25(11):618-628.

30. Anjo D, Santos M, Rodrigues $P$, et al. The benefits of cardiac rehabilitation in coronary heart disease: A gender issue? Rev Port Cardiol. 2014;33(2):79-87

31. Balady GJ, Ades PA, Bittner VA, et al. Referral, enrollment, and delivery of cardiac rehabilitation/secondary prevention programs at clinical centers and beyond: a presidential advisory from the American Heart Association. Circulation. 2011;124(25):2951-2960.

32. Valencia HE, Savage PD, Ades PA. Cardiac rehabilitation participation in underserved populations: minorities, low socioeconomic, and rural residents. J Cardiopulm Rehabil Prev. 2011;31(4):203-210.

33. Grace SL, Gravely WS, Brual J, et al. Contribution of patient and physician factors to cardiac rehabilitation enrollment: a prospective multilevel study. Eur J Cardiovasc Prev Rehabil. 2008;15(5):548-556.

34. Davies P, Taylor F, Beswick A, et al. Promoting patient uptake and adherence in cardiac rehabilitation. Cochrane Database Syst Rev. 2014;25(6):CD007131

35. Taylor R, Dalal H, Jolly K, et al. Home-based versus centre-based cardiac rehabilitation. Cochrane Database Syst Rev. 2010;20(1):CD007130.

36. Ades PA, Pashkow FJ, Nestor JR. Cost-effectiveness of cardiac rehabilitation after myocardial infarction. $J$ Cardiopulm Rehabil. 1997;17(4):222-231.

37. Oldridge N, Furlong W, Feeny D, et al. Economic evaluation of cardiac rehabilitation soon after acute myocardial infarction. Am J Cardiol. 1993;72(2):154-61.

38. Briffa TG, Eckermann SD, Griffiths AD, et al. Cost-effectiveness of rehabilitation after an acute coronary event: a randomised controlled trial. Med J Aust. 2005;183(9):450-455.

39. Yu CM, Lau CP, Chau J, et al. A short course of cardiac rehabilitation program is highly cost effective in improving long-term quality of life in patients with recent myocardial infarction or percutaneous coronary intervention. Arch Phys Med Rehabil. 2004;85(12):1915-1922. 